\title{
Transition of care and the impact on the environment of care
}

\author{
Kerrie S. Guerrero, Susan E. Puls, Dorothy A. Andrew \\ The University of Texas Health Science Center School of Nursing, Houston, Texas, USA. \\ Correspondence: Kerrie S. Guerrero. Address: 16811 Spiced Cider Cypress, Texas 77433, USA. Email: \\ ksguerrero@sbcglobal.net \\ Received: January 27, 2014 \\ Accepted: March 28, $2014 \quad$ Online Published: April 16, 2014 \\ DOI : $10.5430 /$ jnep.v4n6p30 \\ URL: http://dx.doi.org/10.5430/jnep.v4n6p30
}

\section{Abstract}

An unplanned readmission to the hospital within 30 days of discharge is seen as a failure by the healthcare team to appropriately plan for a safe and effective discharge to the next level of care. According to The Center for Medicare \& Medicaid Services (CMS), the national average readmission rate in 2012 was $18.4 \%$. As CMS shifts to a pay for performance strategy, a readmission rate higher than the national average for specific disease processes will result in a financial penalty. Many organizations have identified the need to improve the current discharge planning processes and to provide patients with a safer transition to the next level of care to prevent readmissions. Evidence demonstrates that there is value in reconfiguring the current discharge processes toward interventions that demonstrate a reduction in readmission rates. The discharge process should incorporate a multidisciplinary, multicomponent transition of care intervention that starts while the patient is in the hospital and continues with some type of home-care follow-up. Transition of care is a relatively new term that is used to describe a set of interventions designed to coordinate a patient's care during the movement between healthcare settings. Implementing a well-designed transition of care program allows hospitals to provide a safe Environment of Care to patients during their care transitions. Environment of Care is a term coined by The Joint Commission that is used to describe the environment in which the patient is being cared for. The term usually involves three components: the people, the equipment and tools, and the building. As healthcare changes, it will become increasingly important to provide patients with care management throughout the continuum of care, which means thinking of the patient's Environment of Care in much broader terms. How do transition of care processes affect the patient's Environment of Care? Does a well-implemented transition of care program lead to a positive impact in the patient's overall Environment of Care? This article provides an overview of how implementing a formalized transition of care process can lead to a safer Environment of Care.

\section{Key words}

Transition of care, Environment of care, Discharge process

\section{I ntroduction}

It is no secret that patients being cared for in today's acute care setting have higher acuity and spend less time in the hospital. As patients transition from the acute care setting to the next level of care, they become increasingly vulnerable to adverse events than can lead to an unplanned readmission to the hospital. Hospitals must have a clearly defined process 
that helps the patient and the patient's family safely transition from one healthcare setting to the next. Unfortunately, when patients are discharged from the hospital, they can leave with unclear instructions regarding their medications and no follow-up appointments. When a patient is discharged without crucial information, it can lead to the patient being readmitted. An unplanned readmission to the hospital within 30 days of discharge is seen as a failure by the healthcare team to appropriately plan for a safe and effective transition to the next level of care. Common causes of readmission include medication errors from poorly reconciled medications, inability to access needed care, lack of self-care skills, lack of follow-up knowledge and lack of financial resources.

Healthcare organizations are beginning to pay more attention to the transition of care process as a result of the new pay for performance healthcare system created by the Center for Medicare \& Medicaid Services (CMS), as well as the increased cost of poorly developed transition of care programs. Many hospitals and professional organizations have put processes in place in attempts to decrease the 30-day readmission rate. Despite current efforts, however, the all-cause readmission rate within 30 days for Medicare patients in 2011 remained high at a national average of $19.3 \%{ }^{[1]}$. According to CMS, the national average readmission rate in 2012 dropped slightly to $18.4 \%{ }^{[2]}$. The benefits and challenges of a transition of care program can be seen in both the capitated healthcare plan as well as the fee-for service environment ${ }^{[3]}$. The capitated healthcare plans allow the flexibility to coordinate care across the continuum, but such programs must change their way of thinking beyond the traditional Environment of Care in the hospital setting and begin to think about the healthcare environment in a variety of different settings, including the patient's home. The fee-for-service environment offers financial incentives for high-quality care but is often operating at capacity. A well-designed transition of care program allows hospitals that operate in this environment to safely transition patients sooner to another level of care. This article provides an overview of how implementing a formalized transition of care process in an adult acute care facility can lead to a safer Environment of Care for the patient and the patient's family.

\section{The meaning of transition of care and environment of care}

Transition of care is defined as a set of interventions designed to coordinate care during the patient's movement between healthcare settings. The process is intentional, clearly defined with expectations and accountability, and focuses on the needs of the patient and the caregiver ${ }^{[4]}$. For example, a patient enters the acute care hospital with an acute or chronic illness. Upon the patient's discharge from the acute care facility, the patient might be transferred to either a skilled nursing facility or a rehabilitation facility in which care is provided by a different healthcare team. Finally, the patient is discharged home where care might be provided by a home health nurse. Each time a patient moves from one healthcare team or healthcare setting, it is considered a transition of care. With patient acuity increasing in an already complex healthcare system, it is important for organizations to have a clearly defined transition of care program that provides coordination as patients transfer between different healthcare settings.

Environment of Care is a term coined by The Joint Commission and is used to define the environment in which patients are cared for. The Environment of Care consists of three basic components: the building or space where the care occurs, the equipment used to provide care, and the people involved in the care, including the patients and their caregivers. To provide a safe Environment of Care, hospitals must maintain safe conditions for patients, maintain an environment that is sensitive to patient needs, and minimize any situation that does not support a healing environment.

What does the Environment of Care have to do with a formalized transition of care process? The Environment of Care is typically thought of as the hospital brick and mortar building. As healthcare changes and it becomes increasingly important to provide patients with care management throughout the continuum of care, healthcare organizations will need to think of the Environment of Care in broader terms. What if we looked at the Environment of Care as a continuum, which continues after the patient is discharged from the acute care facility? As organizations begin to put transition of care processes in place, how do these processes affect the patient's Environment of Care? Does a well thought out formalized 
transition of care process have positive impacts on the patient's overall Environment of Care, which goes beyond the brick and mortar buildings?

\subsection{People}

People are the first component within the Environment of Care. The people typically referred to in the Environment of Care definition are the healthcare workers, patients, family members, and anyone else who interacts with or in the patient's environment. During transitions of care, the patient is at increased risk for adverse events. Many care transitions are unexpected and sudden, which leave patients and their families with questions and no knowledge of what to expect next. While patients are in the hospital, they are dependent on the healthcare team to address their needs. When patients are discharged home, they are suddenly expected to manage their own care and recovery. The current discharge process at many organizations leaves much to be desired from the patient's point of view. The discharge process typically includes each discipline providing education for patients with a paper copy of instructions. Case managers and social workers may be available to provide discharge planning for some patients, but in many cases no formal readmission risk assessment is completed and no process exists to identify which patients are at a higher risk for readmission. The patient's primary nurse assumes the responsibility to complete the final check of each discipline and provides documents on diagnosis, medication reconciliation, and follow-up care. The patient leaves the hospital with a mass of papers and instructions that are usually provided on the day of discharge and without any true confirmation of understanding. The discharge process described does not include a multidisciplinary team and it leaves the patient with unanswered questions and unclear follow-up expectations. Yet this is the discharge process at many healthcare organizations. Very few organizations have formalized processes in place that help to coordinate the patient's care across the healthcare continuum.

A formalized transition of care process provides a multidisciplinary approach to the discharge process. When a hospital introduces a formalized transition of care process in which discharge planning truly begins on admission, it allows for the people involved in the patient's Environment of Care, such as the healthcare team, the patient, and the patient's caregiver, to be involved in the process early. As a result, the patient has clear expectations before the day of discharge. The people involved in a formalized transition of care process may include the following: the case manager, the social worker, the primary nurse, the physician, and a discharge coach. The formalization of the process allows the hospital to set clear expectations for each person involved in the transition of care process and therefore positively impacts the patient's smooth transition to the next level of care. Figure 1 is an example of a process map based on the Coleman ${ }^{[3]}$. Transition of Care Model.

This process map provides an example of how a formalized transition of care process might look. This process requires the primary nurse to complete a formal readmission risk assessment within 24 hours of the patient being admitted to the acute care facility. The first decision denoted by a diamond shape in the process map is whether the patient is scored as having a high risk for readmission on the initial readmission risk assessment. If the answer is yes, then the primary nurse will generate a consult for the case manager to see the patient. The case manager will be expected to consult with all patients and their families for patients with a high risk score for readmission on the initial risk assessment tool. This step allows the case manager to initiate a consult with the patient earlier in the acute care hospital stay. The case manager will then complete a more in-depth risk assessment that provides information on whether the patient will require a discharge coach. The second decision denoted by a diamond within the process map is whether the patient will be discharged home. The discharge coach process is labor-intensive and it should be used for patients who are being discharged from the acute care facility to their homes. Patients who are discharged to long-term acute care facilities, rehabilitation facilities, or skilled nursing facilities have employees at the facility to continue working with the patient's transition of care after the acute care stay. If the patient scores at risk for readmission on the second more in-depth screening, and the patient is being discharged home, then a discharge coach will be assigned to the patient. The discharge coach can be the case manager or another member of the patient's care team who assumes the responsibility of facilitating discharge planning with a goal of achieving a safe discharge for the patient. The goal is for all patients discharged home to receive clear discharge 
instructions, a correct medication reconciliation document, a follow-up appointment to the next level of care provider prior to discharge, self-care information, and any follow-up names and contact information the patient may need after discharge.

In summary, the "people" element of the Environment of Care definition includes all people who play a role in minimizing risks to the patient. The people involved in a formalized transition of care process play a similar role. The transition of care program is a well-defined effort by the healthcare team to ensure that the patient has a safe transition to the next level of care while minimizing the risk of an unplanned readmission after discharge. It would only make sense that formalization of the process allows the hospital to set clear expectations for each person involved in the transition of care process and therefore positively impacts the patient's smooth transition to the next level of care.

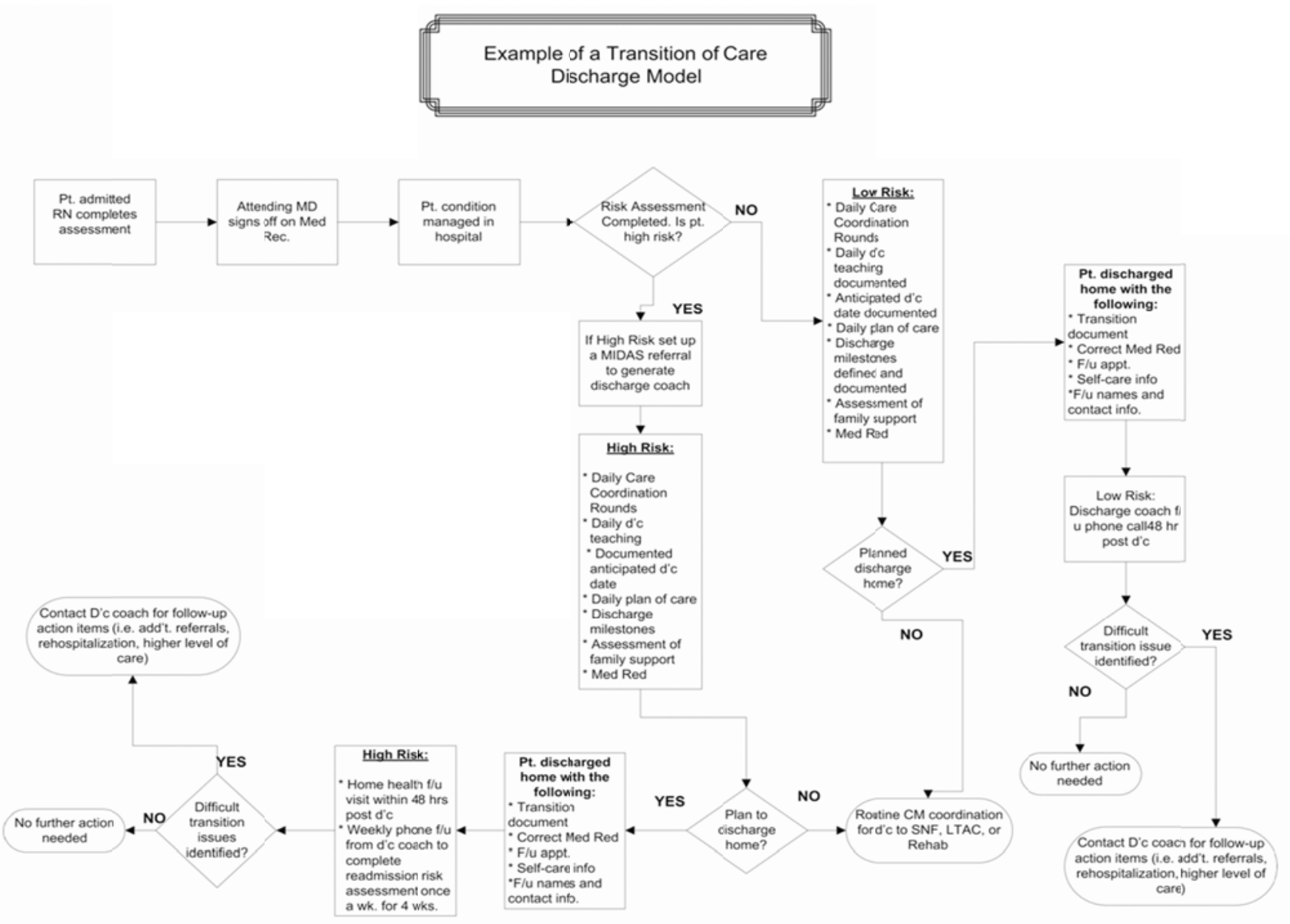

Figure 1. A Transition of Care Discharge Model. Abbreviations: add't, additional; CM, case manager; d'c, discharge; f/u, follow-up; LTAC, long-term acute care; Med Rec, ; Med Rd, ; MIDAS, ; Pt, patient; SNF, skilled nursing facility

\subsection{Equipment and tools}

Equipment and tools are the second component in the Environment of Care. Typically, when thinking about the Environment of Care, healthcare providers think of things like CAT scans, vital sign machines, and glucose monitors. When applying the Environment of Care to a transition of care process, one might think of the equipment and tools as things such as a readmission risk assessment, an accurate list of medications upon discharge, and equipment or tools that can be used to follow up with the patient after discharge from the acute care setting. It is the healthcare organization's duty to provide the healthcare team, the patients, and their families with the appropriate equipment and tools to facilitate a safe transition of care. 
A formal readmission risk assessment tool is vital to a successful transition of care program. The readmission risk assessment allows the hospital to identify the patients at risk for readmission and to intervene early in the patient's hospital stay. Hospitals can purchase software such as a formal readmission risk assessment program, which allows the hospital to clearly identify which patients are at an increased risk of readmission after discharge. Formalized transition of care programs are resource-intensive and often expensive to operate. The risk assessment tools allow the hospital to focus resources on those patients who are more likely to be readmitted within 30 days of discharge. Ideally, the risk assessment would provide information early enough in the patient's hospitalization to trigger the transition of care interventions. A variety of readmission risk assessment tools are available. Coleman developed a 20-item tool that aims to predict readmission to the hospital ${ }^{[5]}$. Hospitals must pick a tool that is easy to use and that works well within the workflow of the organization.

A good medication reconciliation process at discharge is another tool that helps the patient achieve a smooth transition of care out of the acute care setting. Patients tend to leave the hospital confused as to what medications they should take at home. Many times during a hospitalization, physicians will adjust the dosage of a patient's medication or change the medication completely in order to effectively manage the patient's acute symptoms. The patient is then discharged with unclear instructions regarding what medications to take at home. A correct, concise, and easy to follow medication list provides the patient with a tool upon discharge that enables safe self-management of medications at home thus decreasing the likelihood of the patient being readmitted owing to a lack of understanding of post-discharge medications.

Appropriate equipment to follow up with the patient after discharge is also important in a formalized transition of care program. Some organizations have incorporated the use of equipment such as phone calls and video phones as ways to follow up with patients after discharge. Research indicates no significant difference in the effectiveness of either method in preventing readmissions ${ }^{[6,7]}$. Hospitals will need to give some consideration to the complexity of new equipment. Some older patients may have more difficulty with newer technology such as video phones or telemedicine processes.

Providing the healthcare team, the patient, and the family with the appropriate equipment and tools is vital to creating a safe Environment of Care during the patient's transition to the new care setting. In the transition of care process, a readmission risk assessment tool that is completed upon admission, a correct and concise medication list upon discharge, and a clearly defined telephone follow-up process are all examples of some of the equipment and tools that can be used to help create a successful transition of care program. Some or all of the tools discussed can be used during the patient's acute care stay to ensure that the patient is prepared for the transition to the next level of care.

\subsection{Building}

The third component of the Environment of Care is typically the building or space in which the care is taking place. The building or space includes all processes that take place within the building or space that protect patients and lead to safer patient care. The current discharge process being used in many acute care facilities stops at discharge. Once the patient is discharged from the hospital, the patient is on his or her own to perform self-care and follow-up appointments. In 2012, CMS reported that the national average readmission rate was $18.4 \%{ }^{[2]}$, which means the current discharge processes in many healthcare organizations continue to fail to provide millions of patients with a safe transition of care each year. What if healthcare defined the "building" in which the patient receives care as more than the brick and mortar of today's acute care facilities? How would a change in the way we think about the environment in which we provide care affect the quality of care provided to patients?

As healthcare changes and reimbursement is tied to 30-day readmission rates and pay for performance measures, healthcare organizations will need to think about the way the healthcare environment is defined. The new way to think about the healthcare environment must go beyond the brick and mortar of acute care facilities. In fact, research shows that an effective formalized transition of care program should not stop at discharge ${ }^{[7-12]}$. If the patient is discharged home and falls within the high risk of readmission category, then specific steps should be in place to help to ensure that the patient 
undergoes a safe transition of care back into the home. The patients who are at high risk for readmission should receive a visit from a home health nurse within 48 hours of discharge. The home health nurse can perform a patient assessment, help with any self-care deficits, and answer any questions the patient may have after discharge from the hospital. Another intervention that can be performed after discharge to minimize the risk of the patient having an unplanned readmission is a weekly phone call from the discharge coach for up to 4 weeks after discharge. The discharge coach can complete the readmission risk assessment at each interaction with the patient and assess how the patient is self-managing his or her care and whether additional follow-up care is needed. Healthcare organizations must begin to think in broader terms when thinking about the building where care is provided. When thinking about the building aspect within the Environment of Care, hospitals must begin to think of the patient's home as an extension of themselves. When hospitals think of the building in this way, it allows them to create processes that go beyond the brick and mortar of the facility to provide a safe transition of care for the patient.

\section{Summary}

Hospitals are still trying to figure out creative ways to decrease their 30-day readmission rate. An advantage to implementing a transition of care process is that it provides a systematic process that focuses on a safe and effective discharge process, accurate medication reconciliation, and clear follow-up instructions. When looking at the transition of care process through the Environment of Care lens, the two go hand in hand. A formalized transition of care process leads to a safer Environment of Care for the patient. Transition of care processes tend to be labor-intensive and can cost organization thousands of dollars. It is important for hospitals to identify the patients at high risk for readmission and to focus their efforts on these patients. A well thought out implementation plan, patient education that promotes self-care, caregiver education, and support and constant communication and follow-up with the patients will help to create a successful transition of care program.

\section{Conclusion}

At the time of hospital discharge, many patients are at an increased risk for adverse events from the combination of shorter stays, increased severity of illness, and more complex discharge plans ${ }^{[13]}$. It is every hospital's responsibility to ensure that patients are taken care of in a safe environment. Many organizations have identified the need to improve the current discharge planning processes and to provide patients with a safer transition to the next level of care. Public reporting and financial penalties tied to high 30-day readmission rates are forcing organizations to think about the Environment of Care differently and to seek innovative ways to improve the quality of care ${ }^{[14,15]}$. Research has shown that a formal transition of care process has the potential to improve the patient's Environment of Care. Organizations must start to think about the Environment of Care as the care continuum, which goes beyond the patient's discharge from the acute care facility and continues into the patient's home after discharge. Hospitals must focus on all three components within the Environment of Care when implementing a formalized transition of care process. Developing and implementing a formal transition of care process that involves an interdisciplinary team including the patients and caregivers must become a hospital priority. It is time for nurses to take the lead in developing and implementing formalized transition of care processes within their organizations to ensure that patients are provided high-quality discharge processes that lead to a safer Environment of Care.

\section{References}

[1] Centers for Medicare and Medicaid Services. MedPAR data [cited 2014 Jan 21]. Available from: http://www.cms.gov/Research-Statistics-Data-and-Systems/Statistics-Trends-and-Reports/MedicareFeeforSvcPartsAB/MEDPA R.html. 
[2] Gerhardt G, Yemane A, Hickman P, Oelschlaeger A, Rollins E, Brennan N, Centers for Medicare \& Medicaid Services. Data shows reduction in Medicare hospital readmission rates during 2012. MMRR. 2013; 3(2):E1-12. Available from: http://www.cms.gov/mmrr/Downloads/MMRR2013_003_02_b01.pdf. http://dx.doi.org/10.5600/mmrr.003.2013;02.b01

[3] Coleman EA, Fox PD. One patient, many places: Managing healthcare transitions, part i. Ann Longterm Care. 2004; 12 (9): $25-32$.

[4] Boling PA. Care transitions and home health care. Clin Geriatr Med. 2009; 25:135-48. PMid:19217498 http://dx.doi.org/10.1016/j.cger.2008.11.005

[5] Coleman EA, SmithJD, Frank JC, Min SJ, Parry C, Kramer AM. Preparing patients and caregivers to participate in care delivered across settings: The care transitions intervention. J Am Geriatr Soc. 2004; 52(11): 1817-25. PMid:15507057 http://dx.doi.org/10.1111/j.1532-5415.2004.52504.x

[6] Wakefield BJ, Ward MM, Holman JE, Ray A, Scherubel M, Burns TL, et al. Evaluation of home telehealth following hospitalization for heart failure: A randomized trial. Telemed J E Health. 2008; 14(8): 753-761. PMid:18954244 http://dx.doi.org/10.1089/tmj.2007.0131

[7] Cardozo L, Steinberg J. Telemedicine for recently discharged older patients. Telemed J E Health. 2010; 16(1): 49-55.

[8] Aguado O, Morcillo C, Delas J, Rennie M, Bechich S, Schembari A, et al. Long-term implications of a single home-based educational intervention in patients with heart failure. Heart Lung. 2010; 39(6 Suppl): S14-22. PMid:20598745 http://dx.doi.org/10.1016/j.hrtlng.2010.04.010

[9] Bull MJ. Use of formal community services by elders and their family caregivers 2 weeks following hospital discharge. J Adv Nurs. 1994; 19(3): 503-8. PMid:8014311 http://dx.doi.org/10.1111/j.1365-2648.1994.tb01113.x

[10] Coleman EA, Smith JD, Raha D, Min SJ. Posthospital medication discrepancies: Prevalence and contributing factors. Arch Intern Med. 2005; 165(16): 1842-1847. PMid:16157827 http://dx.doi.org/10.1001/archinte.165.16.1842

[11] Robertson KA, Kayhko K. Cost analysis of an intensive home follow-up program for first-time post-myocardial infarction patients and their families. Off J Can Assoc Crit Care Nurs. 2001; 12(4): 25-31. Available from: http://www.crd.york.ac.uk/crdweb/ShowRecord.asp?LinkFrom=OAI\&ID=22002007552

[12] Sinclair AJ, Conroy SP, Davies M, Bayer AJ. Post-discharge home-based support for older cardiac patients: A randomised controlled trial. Age Ageing. 2005; 34(4): 338-343. PMid:15955757 http://dx.doi.org/10.1093/ageing/afi116

[13] Chugh A, Williams MV, Grigsby J, Coleman EA. Better transitions: improving comprehension of discharge instructions. Front Health Serv Manage. 2009; 25(3): 11-32. PMid:19382514

[14] Fung CH, Lim YW, Mattke S, Damberg C, Shekelle PG. Systematic review: the evidence that publishing patient care performance data improves quality of care. Ann Intern Med. 2008; 148(2): 111-23. PMid:18195336 http://dx.doi.org/10.7326/0003-4819-148-2-200801150-00006

[15] Colorado Foundation for Medical Care. National Coordinating Center for the Integrating Care for Populations and Communities [Internet]. Available from: http://www.cfmc.org/integratingcare/. 